\title{
Eye Drops, Emulsion in Single-dose Container
}

National Cancer Institute

\section{Source}

National Cancer Institute. Eye Drops, Emulsion in Single-dose Container. NCI Thesaurus.

Code C149499.

Medicinal product consisting of an eye drops emulsion presented in a single-dose container. 